\title{
NiPreps: enabling the division of labor in neuroimaging beyond fMRIPrep
}

Esteban O., Wright J., Markiewicz CJ., Thompson WH., Goncalves M., Ciric R., Blair RW., Feingold FW., Rokem A., Ghosh SS., Poldrack RA.

\section{Introduction}

The current neuroimaging workflow has matured into a large chain of processing and analysis steps involving a large number of experts, across imaging modalities and applications. The development and fast adoption of fMRIPrep [1] have revealed that neuroscientists need tools that simplify their research workflow, provide visual reports and checkpoints, and engender trust in the tool itself. Here we present the NiPreps (NeuroImaging Preprocessing toolS) framework, which extends fMRIPrep's approach and principles to new imaging modalities. The vision for NiPreps is to provide end-users (i.e., researchers) with applications that allow them to perform quality control smoothly and to prepare their data for modeling and statistical analysis.

\section{Methods}

The NiPreps framework (Figure 1) encompasses a wide array of software projects organized into three layers of scientific software:

- Software infrastructure: including quite mature projects such as NiPype [2] and NiBabel; the standard specifications of the Brain Imaging Data Structure (BIDS [3], and BIDS-Derivatives); and some other tools such as NiTransforms or TemplateFlow, under development. These tools deliver low-level interfaces (e.g., data access to images and spatial transforms) and utilities (see Figure 1).

- Middleware: these are utilities that generalize their functionalities across the end-user tools. These utilities cover foundational processing methodologies (e.g., NiWorkflows and SDCFlows), the crowdsourcing of metadata (e.g., MRIQC Web-API [4]), and the support for deep learning models (MRIQC-nets).

- End-user tools such as fMRIPrep: Some existing end-user tools include sMRIPrep (Structural MRI Preprocessing), which lies in between an end-user tool and middleware, as it is involved in higher-level tools such as fMRIPrep. Finally, quality control tools (e.g., MRIQC [5]) to be executed before any preprocessing happens.

End-user NiPreps abide by the following principles:

1. NiPreps only and fully support BIDS and BIDS-Derivatives for the input and output data.

2. NiPreps are packaged as a fully-compliant BIDS-Apps [6], not just in its user interface, but also in the continuous integration, testing, and delivery.

3. The scope of NiPreps is strictly limited to preprocessing tasks.

4. NiPreps are agnostic to subsequent analysis, i.e., any software supporting BIDS-Derivatives for its inputs should be amenable to analyze data preprocessed with them.

5. NiPreps are thoroughly and transparently documented (including the generation of individual, visual reports with a consistent format that serve as scaffolds for understanding the underpinnings and design decisions).

6. NiPreps are community-driven, and contributors (in any sense) always get credited with authorship within relevant publications.

7. NiPreps are modular, reliant on widely-used tools such as AFNI, ANTs, FreeSurfer, FSL, NiLearn, or DIPY [7-12] and extensible via plug-ins. 


\section{Results}

fMRIPrep is the first NiPrep application and has served to pilot the approach. The tool has successfully helped many researchers to preprocess their data while minimizing friction and time-cost, and maximizing the transparency of their research. The tool has demonstrated an appropriate performance on an extense domain of diverse data, as shown by the number of successful runs around the world (see Figure 2). fMRIPrep has engaged a substantial number of researchers, as evidenced in our latest documentation sprint.

\section{Discussion and conclusion}

fMRIPrep has demonstrated that it is possible to alleviate the comprehensive computing knowledge required for functional MRI studies. Leveraging BIDS and Derivatives, it is possible to view preprocessing as an extension to the scanning device. In other words, standardizing preprocessing is a way of creating smart imaging devices that produce data ready for analysis.

\section{References}

1. Esteban, O. et al. fMRIPrep: a robust preprocessing pipeline for functional MRI. Nat. Methods 16, 111-116 (2019).

2. Gorgolewski, K. et al. Nipype: a flexible, lightweight and extensible neuroimaging data processing framework in Python. Front. Neuroinformatics 5, 13 (2011).

3. Gorgolewski, K. J. et al. The brain imaging data structure, a format for organizing and describing outputs of neuroimaging experiments. Sci. Data 3, 160044 (2016).

4. Esteban, O. et al. Crowdsourced MRI quality metrics and expert quality annotations for training of humans and machines. Sci. Data 6, 1-7 (2019).

5. Esteban, O. et al. MRIQC: Advancing the automatic prediction of image quality in MRI from unseen sites. PLOS ONE 12, e0184661 (2017).

6. Gorgolewski, K. J. et al. BIDS Apps: Improving ease of use, accessibility, and reproducibility of neuroimaging data analysis methods. PLOS Comput. Biol. 13, e1005209 (2017).

7. Cox, R. W. \& Hyde, J. S. Software tools for analysis and visualization of fMRI data. NMR Biomed. 10, 171-178 (1997).

8. Avants, B. B., Epstein, C. L., Grossman, M. \& Gee, J. C. Symmetric diffeomorphic image registration with cross-correlation: Evaluating automated labeling of elderly and neurodegenerative brain. Med. Image Anal. 12, 26-41 (2008).

9. Fischl, B. FreeSurfer. NeuroImage 62, 774-781 (2012).

10. Jenkinson, M., Beckmann, C. F., Behrens, T. E. J., Woolrich, M. W. \& Smith, S. M. FSL. NeuroImage 62, 782-790 (2012).

11. Abraham, A. et al. Machine learning for neuroimaging with scikit-learn. Front. Neuroinformatics 8, (2014).

12. Garyfallidis, E. et al. Dipy, a library for the analysis of diffusion MRI data. Front. Neuroinformatics 8,8 (2014). 


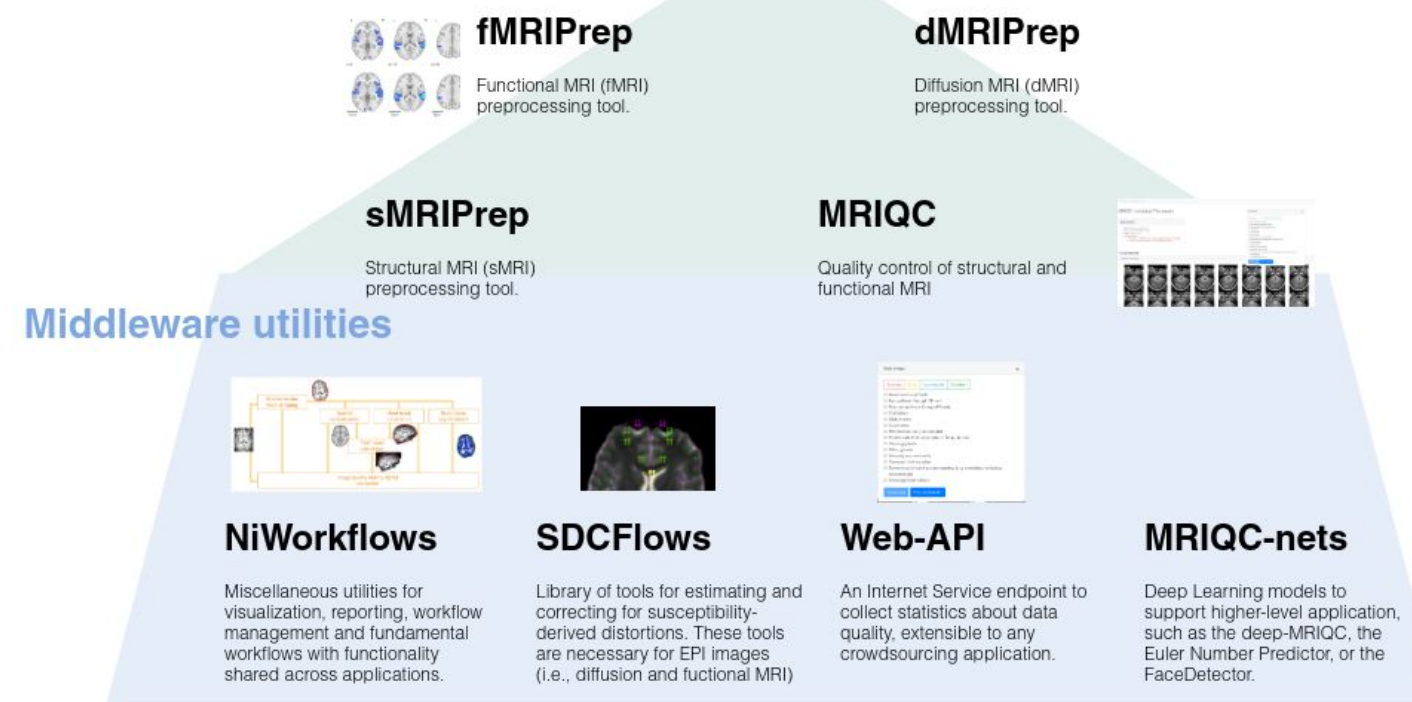

\section{Software Infrastructure}

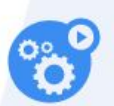

NiPype

The workflow engine supporting the execution graph and run time management (staging tasks,

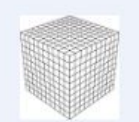

NiBabel

Library supporting the (primaging data formats (PrifTI2).

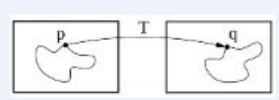

NiTransforms

Library supporting the spatial transformation data formats, and an easy-to-use interface for the

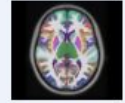

TemplateFlow

A repository and a client tool to allow programmatic access to neuroimaging templates and machines.

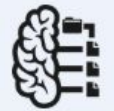

BIDS

The data specification prescribing the formal structure for the neuroimaging data inputs and outputs.

Figure 1. The NiPreps software stack. To ensure the best software engineering practices, and to separately continuously test software, the framework comprehends several striates of tools.
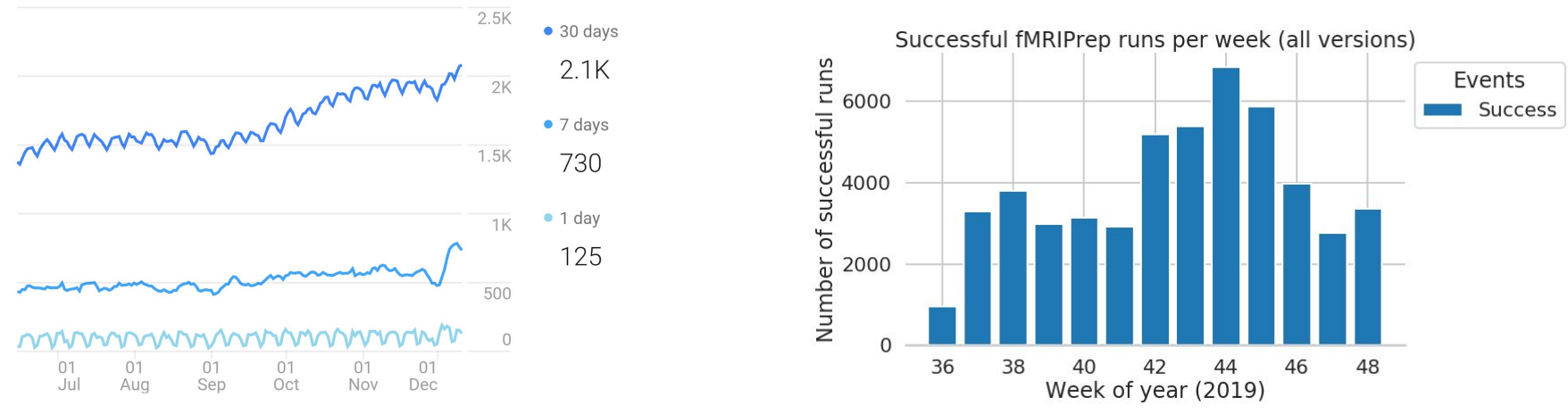

Figure 2. $f M R I P r e p$ is continuously expanding over time. (top) The documentation website has an average of 2,100 monthly visitors, which has steadily increased over time. (bottom) The number of weekly runs is in the order of 3,000 4,000 successful executions. 